\title{
Transpositional Research in the Clinical Process
}

\author{
By Alexander Z. Guiora
}

I

T SEEMS TO ME that two areas are of great concern today to the clinical psychological sciences: the clinical circumstance, both as it affects the welfare of the patient and as a source of our data; and the methodology, or methodologies, most suited, most applicable, to a fruitful and systematic inquiry into that very clinical circumstance.

Let me elaborate on what I mean by "clinical circumstance." The most parsimonious definition of the clinical circumstance, limiting it for the sake of the present discussion to the one-to-one situation, could describe it as a transaction betwen two people who assume clearly distinguishable role models and which transaction is expected to increase, to enhance the well-being of one of the participants (the patient). This definition is broad enough to include paraclinical encounters and general enough to give shelter to everything from psychoanalysis to behavior modification or pastoral counseling.

Nonspecific as it may be, this definition has been and still is, I believe, the cornerstone of the clinical enterprise. What it does not say, however, is of the utmost concern to us. It does not say anything about the transaction itself; it makes no statement at all about the persons interacting. Is this transaction a procedure relatively unaffected by the singularities of the participants? Or is it a unique event totally determined by the idiosyncrasies of the two people involved? $\mathrm{Or}$ is this transaction a continuum between those two poles, and if so, what is the optimal range of the continuum in terms of the stated purpose of the transaction?

A great deal of attention has been paid, especially in the psychoanalytic literature, to the role of the patient in this dyadic relationship. Recent efforts, however, tend to shift the focus to the person of the therapist. Assertions that the therapist's personality does make a difference can be found in a number of sources. There have been several research demonstrations of individual differences attributable to the therapists' personalities. Strupp's studies $^{18,19}$ have clearly demonstrated that there are individual differences between therapists and their approaches to treatment, their ways of conceptualizing, and in general, their attitudes to patients, although he has not shown that these are critical differences for success in therapy. Glover's survey of analytic practice ${ }^{5}$ demonstrated wide differences in what was done and what was thought important among analysts with relatively homogeneous training. Pilowsky and Spear ${ }^{15}$ showed that two psychiatrists seeing the same patients at different times elicited significantly different amounts of talk from the patient when for each the only verbal cue was to say at the

From the Departments of Psychiatry and Psychology, University of Michigan, Ann Arbor, Mich.

Alexander Z. Guiona, Ph.D.: Professor of Psychology, Department of Psychiatry and Psychology; Chief Psychologist, Neuropsychiatric Institute; Member, Center for Research on Language and Language Behavior, University of Michigan, Ann Arbor, Mich. 
beginning: "Would you tell me about the way you feel." Studies showing that untrained therapists can produce significant improvement in patients in some cases more than trained professionals ${ }^{2,3,16}$ suggest that some personal rather than technical factor is involved in at least these changes.

There has been a flurry of studies in the research literature about attitudinal factors, mainly the therapist's liking his patient as a factor in treatment. $\mathrm{Kahn}^{13}$ found that therapists who were more negatively disposed toward patients perceived their dynamics and behavior less accurately, though positive feelings towards patients did not lead to significantly more accuracy. Abeles $^{t}$ failed to find a relationship between therapist's liking for patients and his empathic understanding. McNair et al. ${ }^{14}$ did not find clear-cut evidence that liking for the patient increased likelihood of staying in treatment, though there was suggestive evidence that therapists with strong liking for their patients retained higher proportions of both patients who tend to leave therapy prematurely and those who would tend to stay. Stoller ${ }^{17}$ reversed the perspective, studying the likeability of patients as judged from 2-minute segments of tape and found that more successful patients received a significantly higher mean likeability score. In regard to therapists' interest in their patient specifically, there is much evidence that patients tend to see this as one of the most important factors.

I can cite a number of studies here which conclude, like Board ${ }^{4}$ that interest in the patient by the therapist is a sine qua non of successful therapy, and so on. Studies done by Strupp et al. ${ }^{20}$ found in a sample of patients from primarily psychoanalytically oriented therapy that "therapist's respect and interest" correlated 0.63 with the amount of therapeutic change. A collection of studies with different types of patients and therapy provides convincing evidence, seemingly that patients who find the therapist's interest in them to be a vital factor in their own positive feelings about therapy and its results.

The inquiry into the attitude of the therapist as a factor in the clinical transaction was taken one step further by attemps to list the attitudes which a good therapist should have. Holt and Luborsky ${ }^{12}$ present a summary of the views of a number of writers, especially in the analytic tradition. Most of the attributes looked for are those which any profession would like to characterize its members by: high intelligence, good character, willingness to take responsibility, understanding, and so on. Beyond them there are specific capabilities mentioned such as the ability to take a therapeutic attitude, empathy, warmth, and so on.

I have sketched a cursory overview of current literature only to indicate a trend, i.e., that the transactional process in general and the therapist variable in particular seem to occupy a place of growing importance in the psychological sciences.*

Writing on this issue some years ago I suggested that,

*This limited review of the literature is based on: David Chandler, The Person of the Therapist as a Factor in Psychotherapy, A review of the literature. Unpublished precandidacy paper, The University of Michigan, 1967. 
"Faced with a variety of clinical behaviors, diagnostic and predictive, which do not lend themselves to obvious understanding, we are hard put to provide meaningful formulations to explain these clinical behaviors. In the literature dealing with psychological testing the terms most often used to denote the meaningful grasp of less than obvious clinical data are "inference" and "intution" while in the literature dealing with psychotherapy or psychoanalysis the terms "empathy" and "institutution" are most frequently encountered. A survey of the relevant writings reveals a considerable lack of clarity, if not outright confusion, in the way these terms are used. The overall impression is that these three terms are intended to denote three different kinds of processs but they are used, at times, as synonymous, or one serves as an elaboration of the other. Some clarity of delineation of these terms is needed for they do represent three distinct modes of comprehending clinical data". ${ }^{7}$

I would like to repeat the definitions of inference, empathy and intution as offered earlier:

\footnotetext{
"Inference is a cognitive process of comprehending, characterized by derivation of conclusions from a given set of data or premises in compliance with the rules of Aristotelian logic."7

"Empathy is a process of comprehending in which a temporary fusion of selfobject boundaries as in the earliest pattern of object relation permits an immediate emotional apprehension of the affective experience of another, this sensing being used by the cognitive functions to gain understanding of the other." 7

"Intuition is a mode of comprehending in which external cues normally inadequate for logical judgment and/or prediction give rise to apparently direct immediate and accurate judgment and/or prediction through the mediation of idiosyncratic associations organized according to allological principles."9
}

In the light of these definitions of intuition, empathy and inference certain significant differences may be seen. What constitutes the essential distinction between intuition and empathy is the basic orientation involved in these modes of comprehending. Empathy reaches out toward another person; the intimate sharing of experience that it connotes is made possible by a partial giving up of the self in order to include the other person. In contrast, intuition turns toward the self. The comprehending of another person is, in the intuitive act, a kind of incidental derivative of a narcissistically-oriented process rather than an expression or a resultant of object-directed concern.

Inference too is more object-directed that is intuition, but the aim of this process is based more on the cognitive need for understanding than a deep emotional concern and sensitivity characteristic of the empathic process. The differentiation between inference and intuition is to be found in the nature of the principles underlying the associated connections which lead to the understanding gained. As noted in the definitions given, inference operates by deriving conclusions from presented data in accordance with Aristotelian logic whereas intuition yields its conclusions by means of allological principles.

We now come to the second of the concerns we posited were critical in the psychological sciences today. The question of methodology: what is the best 
way, or best ways, in which we can submit the hypotheses emanating from the clinical circumstance to a systematic and critical examination that will yield lawful relationships between the observed phenomena, reliable and valid predictions about future events, and the results of which lend themselves to public scrutiny.

In posing the problem and describing the desirable characteristics of the solution I am obviously not breaking new ground. The question of methodology, or appropriate methodologies, has pursued in many ways the psychological sciences from their very beginnings. There are obvious difficulties. The scientific method, so critical and fruitful to the development of Western civilization cannot be applied automatically without modifying it, to our data, to the clinical circumstance. This recognition led to varied and, at times, surprising if not always fruitful responses.

On the one extreme it was suggested that if our data do not fit the scientific method, then they are bad data and are outside the realm of scientific inquiry. A corollary and equally extreme position is that if the scientific method does not fit out data, then the scientific method has to be abandoned. Thus an easy and angry dichotomy has been created between the "scientists" and the "practitioners" in the field, neither contributing to nor influencing the other. A dialogue of the deaf ensued, the only comprehensible sounds of which are mutual recriminations and disparagements.

Between these two extreme positions we find a number of attempts to bridge the gap between clinical data and the ill-fitting scientific method. These attempts fall essentially into two categories: reductionist and analogue research.

Reductionism, briefly stated, is an attempt to operationalize the hypothesis generated in the clinical situation so that it lends itself to empirical investigation. The obvious hazard lies in reducing the original question too much so that the ensuing result will border on the trivial. Psychological journals are full of this kind of well-intended exercise in futility.

Analogue research as a strategy recognizes the liabilities of extreme reductionism and attempts to create an assumed analogue to the original clinical circumstance. Some of Edward Bordin's work illustrates this research strategy; e.g., studies where a laboratory-free association exercise is substituted for the original analytic situation. This approach is more promising than the different shades of reductionism but it has its obvious limitations as to the "realness" of the laboratory situation and as to the meaningfulness of the findings.

In reflection, it seems to me that clinical research implies a systematic attempt to gain knowledge about human functioning, in circumstances usually significantly different from those of the laboratory. The nature of the clinical setting imposes limits on the freedom of data collection and manipulation of subject population. Clinical research strategies must be adapted to available, natural sources of information, whereas data collection in most laboratory research can follow the dictates of controlled best design. Further, the subjects of clinical psychological research are, more likely than not, apt to be people in pain, who approach every procedure and intervention with conscious or unconscious expectations and apprehensions and whose integrity 
and human dignity take unconditional precedence over experimental needs and procedures.

The relevance and success of scientific inquiry depend, in the main, on three crucial steps: (a) the recognition or discovery of significant problem areas that need and can be explored, (b) the formulation of clear and appropriate questions in terms that lend themselves to empirical investigation in such a way that the ensuing data will have relevance to the question posed, and (c) the use of appropriate techniques of analysis, both statistical and conecptual, for the evaluation of the information gathered.

The research strategy that we came to rely on in our own work can be briefly described as follows: in face of the built-in liabilities of the reductionist and analogue research we have abandoned attempts to achieve better operationalizations. Instead of that we try to identify another realm of behavior, where the phenomena, first observed in the clinical situation, can be assumed also to exist. That other realm of behavior however, in contradistinction to the original clinical circumstance, is of such a nature that it lends itself readily to manipulation of variables and populations, to rigorous empirical research. I call this strategy transpositional research.

Let me illustrate this approach by a description of my current work in empathy research. In a recent extensive review of the empathy research literature $^{s}$ I have suggested that previous work in this area suffers from two major weaknesses. The definitional formulations used are not specific enough, and more importantly, the way these definitions are operationalized does not allow for a high level of construct validity. In most instances empathy has been operationalized either in terms of therapeutic behavior to be rated and judged by other therapists or in terms of a rather global person-perception model.

Since 1966, my associates* and I at The University of Michigan have been engaged in an effort to develop a research strategy that would correct the weaknesses ascribed to previous research in the field of empathy. The first task then was to operationalize the concept of empathy in such a way that maximum construct validity would be assured, and then to develop an appropriate measure of the operationalized behavior.

The new research strategy that evolved required that the operationalized behavior be outside the psychotherapeutic realm. The hypothesis generated in the clinical circumstance had to be "lifted out" and transposed to a behavioral realm where more rigorous, reproducible, reliable and valid experimentation was feasible. We posited that once a hypothesis generated in the clinical (naturalistic) setting could be tested out in the laboratory (the operationalized behavior), we could then hope to reapply our laboratory findings to the original circumstances.

The behavioral realm chosen was that of language behavior. Language is the most expressive, and most highly developed communication modality and

* The people associated with this research project at one time or another are: L. A. Bosworth, M. A. Brandwin, J. C. Catford, R. Garwood, N. Kalter, H. L. Lane and L. L, Taylor. 
the most essential instrument in the clinical realm. Furthermore, language behavior provides us with a vehicle for reproducible experimentation.

The second half our research strategy formula requires that identical processes be noted in the original and the transposed realms of behavior.

Language behavior is a unique and complex attribute of man, not only in the evolutionary sense, but in the developmental psychological history of each individual. Language behavior arises and evolves within the context of a more general psychological growth. It is reasonable to speculate that even certain structural aspects of language are in part shaped by and express the broader personality context from which they have emerged. ${ }^{11}$

Focusing on second language acquisition, and more particularly on the ability to assimilate nativelike authenticity of pronunciation in a foreign language we have suggested ${ }^{10}$ that empathy may play a significant role in a learner's relative ability to acquire authenticity of pronunciation in a second language.

Thus we have a theory of empathy generated in the clinical circumstance, based on clinical observations, and a realm of behavior outside the psychotherapeutic, namely language behavior, where the presence of the same empathic process can be postulated.

In a series of studies we examined the proposition that research with the empathic process can be pursued by using pronunciation of a second language as its laboratory setting:, $, 10,21,22$

While the specific aim of constructing a unique and direct method of measuring empathy has not yet been achieved in these studies, important and significant relationships between authenticity of pronunciation of a second language and constellations of factors representing the personality variables of empathy and intuition have been found, thus confirming the hypothesis under investigation and the underlying psychological theory. It has been shown that personality characteristics interacting with the interpersonal situation of language learning result in differences in language performance, specifically in the authenticity of pronunciation.

Two constellations of variables representing independent modes of comprehending were identified and each was a significant predictor of a skill in second-language pronunciation. The empathy dimension consisted of a measure of tolerance to anxiety caused by affective stimuli, intelligence necessary for cognitive understanding, a cautious style of responding only to affective cues of the other, and the ability to be involved in emotional experiences. The first two, anxiety and intelligence, are adjuncts to empathic functioning, while the last two, attention to and involvement in emotional expression, are independent components of empathic capacity. The empathy dimension is significantly related to the ability to learn and recall exact details in the pronunciation of second language, accounting for more than half the variance of this skill.

A second mode of comprehending was defined by three unrelated variables and represents the intuitive dimension. Anxiety as a distractor and intelligence as a facilitator are again necessary parts of this dimension; its prime factor is the component of understanding the motives and complexities of interpersonal situations and gaining mastery through self-directed reflection. This dimension 
of intuition is significantly related to the ability to sound fluent and authentic in repeating sentences, accounting for more than half the variance of this skill, but not to the exact pronunciation of precise details predicted by the empathy dimension. ${ }^{21}$

Further studies are under way, the results of which are either not yet in, or not yet completely analyzed. Findings to this date are sufficiently encouraging to anticipate further confirmation of the hypothesized empathy-authenticity association. Such confirmation would imply the possiblity of developing, through further refinement of the techniques discussed, a predictive measure of success in the acquisition of pronunciation authenticity in second language learning. More importantly, and beyond the specific focus of investigation touched upon in these studies, our program of research has enhanced our belief in the heuristic value of systematic empirical exploration of personality variables in language behavior. The operationalizing and testing of clinical psychological concepts in the language laboratory, we believe, can provide the psychological and linguistic disciplines with reciprocal insights and understanding.

Furthermore, while improvement in the measures is clearly desirable, our investigations have demonstrated that empathy and intuition need not be treated as mysterious and unscientific simply because they refer to complex hypothesized internal processes.

In terms of the original concerns relevant to the clinical circumstance and its inquiry it would seem that transpositional research provides a strategy that offers certain advantages over the existing ones. It is conducted without doing violence to any of the principles concerning patient welfare; it is removed from the clinical situation and thus rendered immune to the influence of intervening variables that are cluttering up so much of contemporary clinical research; it discourages atheoretical, correlational studies and enables rigorous reproducible experimentation.

Reproducibility has been a major weakness of most clinical research. Replication of clinical studies usually produces more questions than provides answers. Transpositional research resolves the problems of reproducibility inherent in the clinical situation by stepping outside of it.

No claim is made that we have found the ideal research methodology that will. usher in the millenium of good research in the clinical process. It is maintained however that we have taken a step in the right direction, and that transpositional research has heuristic value for a systematic exploration of the clinical circumstance.

The next step is the conduct of a series of clinical crossvalidational studies. Should such studies yield powerful results, the advantages of transpositional research will have been clearly established.

\section{REFERENCES}

1. Abeles N.: Living for clients-its relationship to therapist's personality and empathic understanding. Amer. Psychol. 19: 522-523, 1964.

2. Anker, J. M., and Walsh, R. P.: Group psychotherapy, a special activity program, and group structure in the treatment of chronic schizophrenics. J. Consult. Clin. Psych. 25:476-481, 1961.

3. Beck, J. S., Kantor, D., and Gelineau, 
V. A.: Follow-up study of chronic psychotic patient "treated" by college case-aid volunteers. Amer. J. Psychiat. 120:269-271, 1963.

4. Board, F. A.: Patients' and physicians' judgments of outcome of psychotherapy in an outpatient clinic. AMA Arch. Gen. Psychiat. 1:185-196, 1959.

5. Glover, E.: The Technique of Psychoanalysis. New York, International Universities Press, 1955.

6. Garwood, R., Guiora, A. Z., and Kalter, N.: Manifest anxiety and perception of micro-momentary expression. Unpublished paper 1970.

7. Guiora, A. Z.: On clinical diagnosis and prediction. Psychol. Rep. 17:779-784, 1965.

8. - : Toward a systematic study of empathy. Compt. Psychiat. 8:375-387, 1968.

9. - Bolin, R., Dutton, C. and Meer, R.: Intuition, a preliminary statement. Psych. Quart. Suppl. 39:110-122, 1965.

10. -, Lane, H. L., and Bosworth, L. A.: An exploration of some personality variables in authentic pronunciation of a second language. In H. L. Lane and E. M. Zale, (Eds.): Studies in Language and Language Behavior, 4:510-514, 1967.

11. Guiora, A. Z., Taylor, L. C., and Brandwin M. A.: A Contribution to the psychology of second language behavior. Proc. XVIth Intern. Congr. of Applied Psych. Amsterdam, Swets and Zeitlinger, 585-588, 1969.

12. Holt, R. D., and Luborsky, L.: Personality Patterns of Psychiatrists. New York: Basic Books, 1958.
13. Kahn, R. K.: Therapist discomfort in two psychotherapies. Diss. Abstr., 17:11819, 1957.

14. McNair, D., Lorr, M., and Callahan, D. M.: Patient and therapist influences on quitting psychotherapy. J. Consult. Clin. Psych. 27:10-17, 1963.

15. Pilowsky, I. and Spear, F. G.: The psychiatrist's role in the interview situation. Amer. J. Psychother. 18 Suppl. 1:174-183, 1964.

16. Poser, E. G.: The effect of the therapist's training on group therapeutic outcome. J. Consult. Clin. 30:283-295, 1966.

17. Stoler, N.: Client likeability; a variable in the study of psychotherapy. J. Consult. Clin. Psych. 27:175-178, 1963.

18. Strupp, H. H.: Patient-doctor relationships: Psychotherapist in the therapeutic process. In Bachrach, A. J. (Ed.): Experimental Foundations of Clinical Psychology. New York: Basic Books, 576-605, 1962.

19. - : Psychotherapists in Action. New York, Grune and Stratton, 1960.

20. -, Wallach, M. J., and Nogan, M.: Psychotherapy experience in retrospect: Questionnaire survey of former patients and their therapists. Psychol. Monogr. 78, No. 1, 1964.

21. Taylor, L. L., Guiora, A. Z., Catford, J. C., and Lane, L. L.: The role of personality variables in second language behavior. Compr. Psychiat. 10:463-474, 1969.

22. -, Catford, J. C., Guiora, A. Z., and Lane, L. L.: Psychological variables and ability to pronounce a second language, Lang. Speech, 1970, (in press). 